\title{
Single molecule detection and underwater fluorescence imaging with cantilevered near-field fiber optic probes
}

\author{
Chad E. Talley, M. Annie Lee, and Robert C. Dunn \\ Department of Chemistry, University of Kansas, Lawrence, Kansas 66045-0046
}

(Received 19 February 1998; accepted for publication 3 April 1998)

\begin{abstract}
Tapping-mode near-field scanning optical microscopy (NSOM) employing a cantilevered fiber optic probe is utilized to image the fluorescence from single molecules and samples in aqueous environments. The single molecule fluorescence images demonstrate both the subdiffraction limit spatial resolution and low detection limit capabilities of the cantilevered probe design. Images taken as a function of tip oscillation drive amplitude reveal a degradation in the resolution as the amplitude is increased. With all cantilevered probes studied, however, a minimum plateau region in the resolution is reached as the drive amplitude is decreased, indicating that the tapping mode of operation does not reduce the optical resolution. Images of fluorescently doped lipid films illustrate the ability of the probe to track small height changes $(<1.5 \mathrm{~nm})$ in ambient and aqueous environments, while maintaining high resolution in the fluorescence image. When the tip is immersed in water $(1.3 \mathrm{~mm})$, the cantilevered NSOM tip resonance, $25-50 \mathrm{kHz}$, shifts approximately $100-150 \mathrm{~Hz}$, the amplitude dampens less than $40 \%$ and the $Q$ factor is reduced from 300-500 to 100-200. (C) 1998 American Institute of Physics. [S0003-6951(98)04022-4]
\end{abstract}

Near-field scanning optical microscopy (NSOM) has been successfully utilized to investigate a wide variety of samples ranging from solid state devices to single molecules. ${ }^{1-5}$ As the technique continues to evolve, developments that improve upon the imaging performance, flexibility, and ease of use continue to appear. As part of this development, our group and others have been exploring the imaging capabilities of cantilevered NSOM tips. ${ }^{6-9}$ Instead of dithering the probe laterally with respect to the sample surface, as is done in the shear-force technique, these probes are oscillated normal to the sample surface in a tappingmode force feedback arrangement, much like tapping-mode AFM.

In a previous report, initial results using cantilevered NSOM tips to image fluorescently labeled rat cortical neurons were presented. ${ }^{7}$ These samples exhibited complex topographies and were selected for the study to demonstrate the ability of the cantilevered probe to track large changes (microns) in sample height, which are often challenging to accurately follow using the shear-force technique. The enhanced surface tracking capabilities, high resolution, and reduction in forces imparted to the sample make the cantilevered probes well suited for imaging biological specimens. However, in spite of these encouraging results, the cantilevered probes do suffer from several disadvantages compared to conventional tips. They are harder to fabricate due to complications arising from the aluminum coating process and they suffer from large bending losses, which dramatically reduce their light throughput efficiency. In general, the cantilevered probes are at least an order of magnitude less efficient than conventional straight NSOM probes fabricated in our lab. This raises concerns about their general utility, especially in low light level applications such as single molecule detection and spectroscopy.

Single molecule spectroscopy is emerging as a powerful tool for probing systems free from the ensemble averaging encountered in bulk samples. ${ }^{1,2,5}$ As such, the ability to fluorescently image single molecules using new NSOM tip de- signs becomes an important criterion for evaluation. As stated earlier, the large bending losses encountered with the cantilevered NSOM probe raise concerns about their ability to deliver enough light for these types of applications. However, because the reduction in throughput results from bending losses that occur far from the tip aperture, more light can simply be coupled into the probe to compensate for the large losses. ${ }^{7}$ Therefore, comparable excitation powers are attainable with the cantilevered tip, since the limiting factor on output power for both aluminum coated tip designs arises from local heating effects occurring near the tip aperture. ${ }^{10,11}$ This is illustrated in Fig. 1 which shows the NSOM fluorescence image of single molecules taken using a cantilevered near-field tip.

Shown in Fig. 1 is a $10 \mu \mathrm{m} \times 10 \mu \mathrm{m}$ NSOM fluorescence image of single dye molecules dispersed in a lipid film. Monolayers consisting of $L$ - $\alpha$-dipalmitoylphosphatidylcholine (DPPC) (sigma), doped with $1 \times 10^{-6} \mathrm{~mol} \%$ fluorescent 1, 1'-dioctadecyl-3,3,3',3'-tetramethylindocarbocyanine perchlorate $\left(\mathrm{diIC}_{18}\right)$ (Molecular Probes), were deposited onto a mica surface at a surface pressure of $15 \mathrm{mN} / \mathrm{m}$ utilizing the Langmuir-Blodgett technique (Model 611, Nima Technology). The dye was incorporated into the lipid monolayer to decrease the effects of photobleaching. Each peak in Fig. 1 represents the fluorescence from a single diIC $_{18}$ molecule and has a FWHM of approximately $110 \mathrm{~nm}$, which reflects the diameter of the NSOM probe used in the imaging. The $256 \times 256$ pixel image was collected with a pixel dwell time of $4 \mathrm{~ms}$ and shows a signal-to-noise ratio comparable to single molecule images collected in our lab using straight NSOM probes.

The fluorescence image shown in Fig. 1 demonstrates the single molecule detection limits attainable with the less efficient cantilevered NSOM probes. Another concern when using cantilevered NSOM probes operating in tapping mode is the possibility of degradation in the optical resolution due to the oscillation of the tip normal to the sample surface. It is important in the tapping-mode arrangement to keep the 


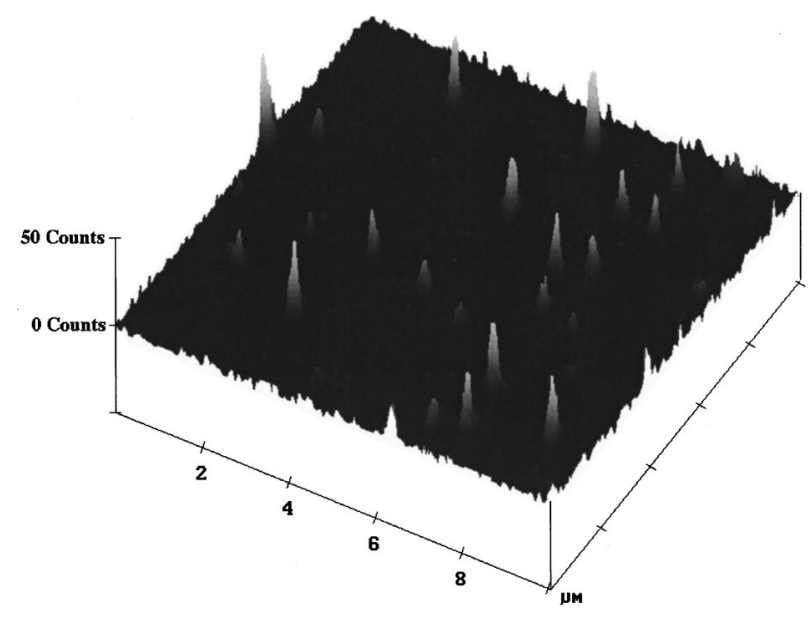

FIG. 1. $10 \mu \mathrm{m} \times 10 \mu \mathrm{m}$ near-field fluorescence image of single diIC $\mathrm{C}_{18}$ molecules in a DPPC lipid monolayer, taken using a cantilevered fiber optic NSOM probe.

maximum vertical tip oscillation distance smaller than the NSOM tip aperture radius. Minimizing this distance ensures that the aperture remains within the collimation region and will, therefore, maintain the highest possible resolution in the fluorescence image. To characterize this effect, NSOM fluorescence images were taken of a dye doped lipid film as a function of tip oscillation drive amplitude. Figures 2(a) and 2(b) show the same film region imaged using tapping drive amplitudes of $500 \mathrm{mV}$ and $3 \mathrm{~V}$, respectively. Higher molecule coverages were used in these experiments to compensate for photobleaching arising from the repeated scanning of the same area. At the lower tapping drive amplitude, Fig. 2(a), high resolution fluorescence features are observed which become degraded as the drive amplitude is increased to $3 \mathrm{~V}$, Fig. 2(b). While the oscillation amplitude is tip dependent and difficult to quantify, with all tips studied, a minimum plateau in the near-field fluorescence spatial resolution was reached as the drive amplitude was reduced. This indicates that, at low drive amplitudes, the cantilevered tips remain within the collimation zone and resolution is not compromised by the vertical tapping motion.

In addition to the single molecule detection limits illustrated above, and the ability to track the complicated topography in biological specimens shown previously; ${ }^{7}$ the study of structure-function relationships in biological samples requires that the NSOM probes have the ability to detect small changes in surface height for samples in both ambient and aqueous environments. These capabilities are illustrated in Fig. 3 which shows near-field fluorescence and force images of DPPC lipid films on a mica substrate both dry, a and b, and under water, $\mathrm{c}$ and $\mathrm{d}$. The supported films were fabricated by the vesicle fusion method described by Kalb and co-workers $^{12}$ and doped with 0.25 mol $\%$ diIC $_{18}$, which selectively partitions into the liquid-like lipid phase and serves as a marker for the domain structures present in the lipid film.

In Figs. 3(a) and 3(b), the simultaneously measured NSOM fluorescence and tapping-mode force images, respectively, of a $12.5 \mu \mathrm{m} \times 12.5 \mu \mathrm{m}$ region of a dry lipid film are shown. The fluorescence structure correlates with the small height changes observed in the force image. The lower to-
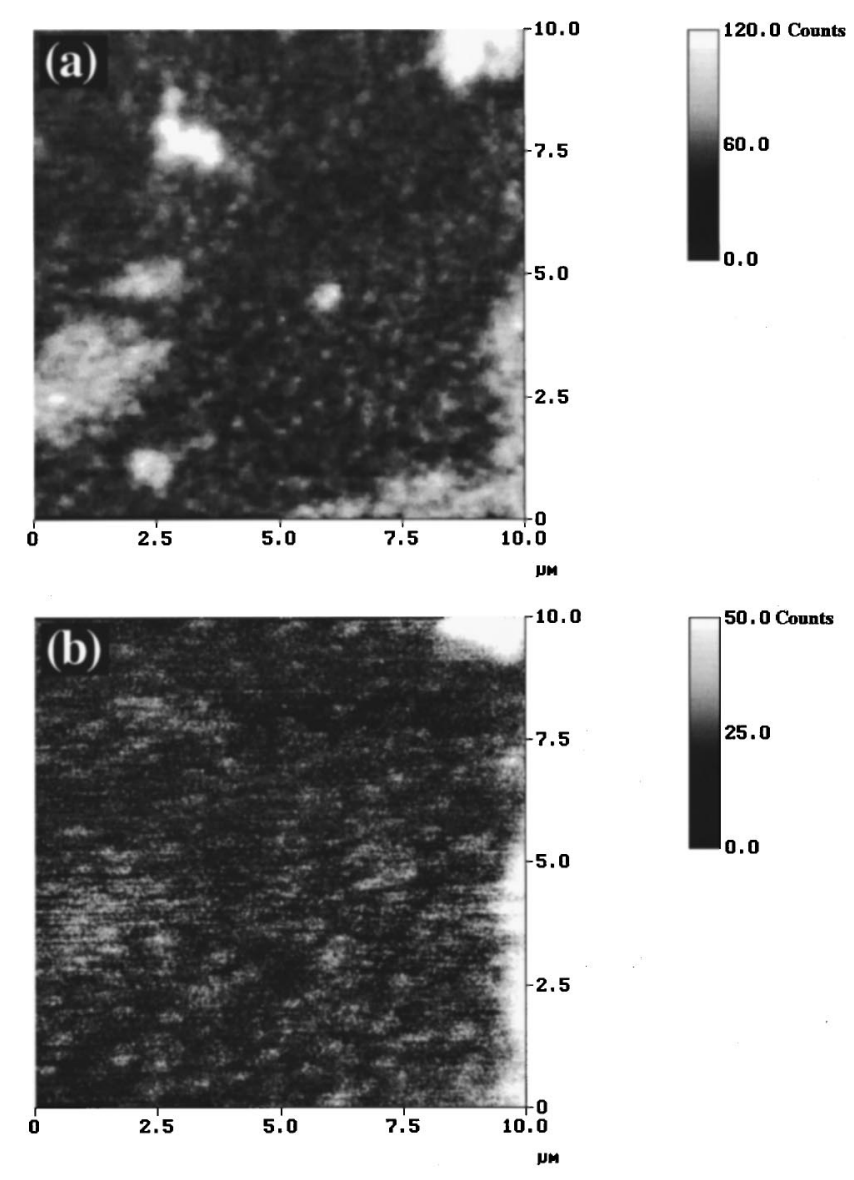

FIG. 2. $10 \mu \mathrm{m} \times 10 \mu \mathrm{m}$ near-field fluorescence images of single diIC 18 molecules in a DPPC monolayer, taken at tip oscillation drive amplitudes of (a) $500 \mathrm{mV}$ and (b) $3 \mathrm{~V}$.

pography regions in the force image appear brighter in the fluorescence image, indicative of the selective partitioning of the probe molecule into the liquid-like domains of the lipid film. The height changes associated with the coexisting lipid phases are less than $1.5 \mathrm{~nm}$, which are easily detected in the force image. Figures 3(c) and 3(d) show corresponding NSOM fluorescence and tapping-mode force images, respectively, of a similar lipid film under water. The $7.5 \mu \mathrm{m} \times 7.5$ $\mu \mathrm{m}$ underwater images of the lipid film show less correlation between the fluorescence and force images. Fluorescence is observed from both high topography and low topography regions of the lipid film which reflects the formation of multilayer structures in the aqueous environment. These images show that minimal degradation in the resolution of either the fluorescence or force images is introduced by the aqueous surroundings.

As pointed out previously, the performance characteristics for cantilevered NSOM probes operating in the tappingmode arrangement are usually less affected by the aqueous surroundings than with straight probes operating in shearforce feedback. ${ }^{6}$ For a water layer of $1.3 \mathrm{~mm}$, we typically find that the amplitude of the resonance is damped less than $40 \%$ and the resonance frequency, $25-50 \mathrm{kHz}$, shifts approximately $100-150 \mathrm{~Hz}$. The tip $Q$ factor, which is typically $300-500$, is reduced to $100-200$ in the aqueous environment. We also investigated the effects of probe heating in both dry and aqueous surroundings by monitoring changes in the approach (force) curve as a function of tip output 

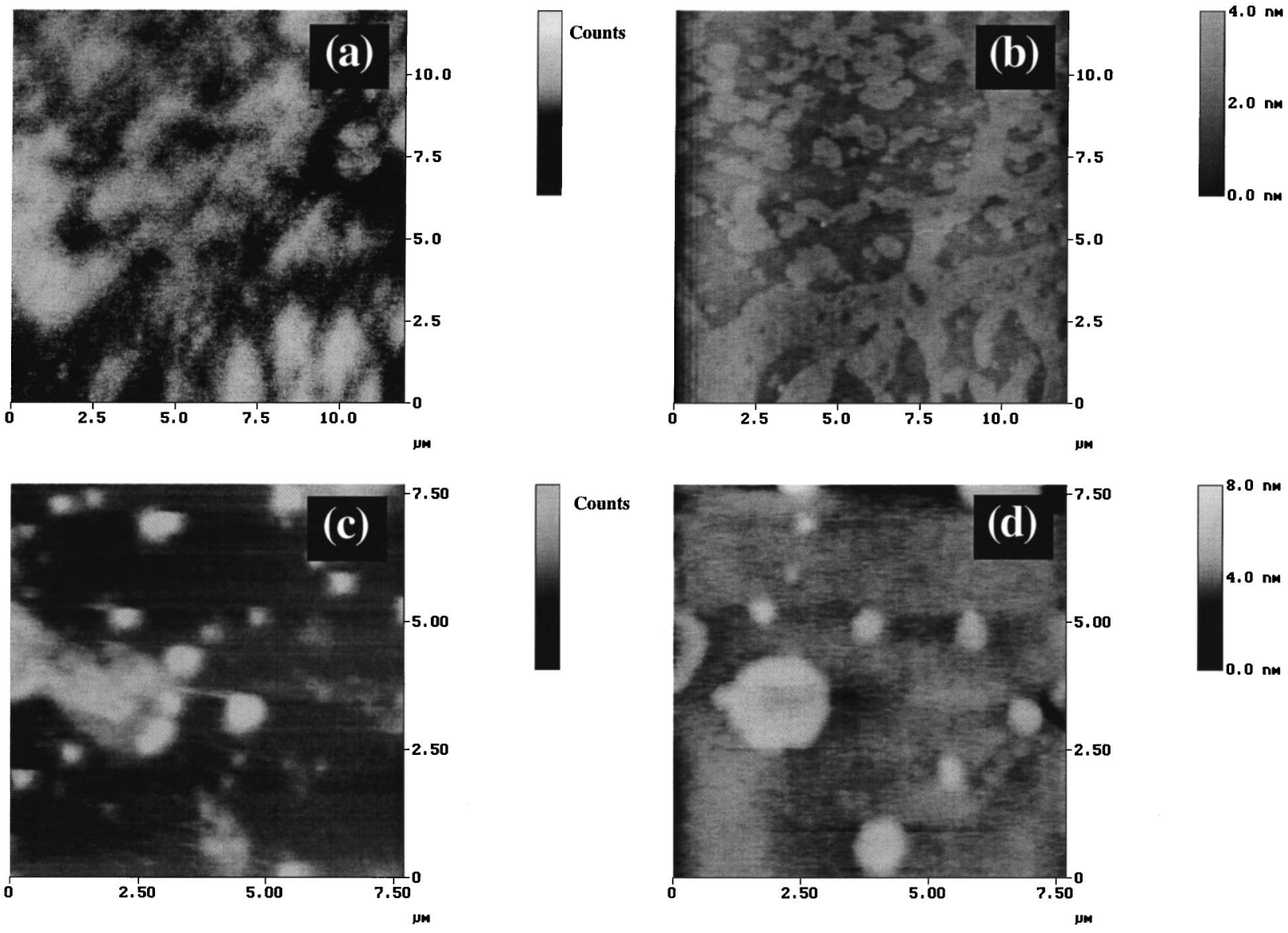

FIG. 3. Near-field fluorescence and topography images of DPPC/diIC ${ }_{18}$ films in air, a and b, and under aqueous conditions, $\mathrm{c}$ and d. In air, the fluorescence from the liquid-like lipid phase (a) is correlated with the low topography regions observed in (b) the force image. Under aqueous conditions, the fluorescence (c) and force (d) force images are less correlated and indicate the formation of multilayer films. In both air and aqueous surroundings, the tapping-mode force images are sensitive to height changes in the film of less than $1.5 \mathrm{~nm}$.

power. ${ }^{10,11,13,14}$ These changes were also compared to straight NSOM tips to further quantify the differences between the two probe designs. In general, both expansions and contractions of the tips were observed in the approach curves as the output power was increased to $10 \mathrm{nW}$. The changes ranged in magnitude from 0 to $250 \mathrm{~nm}$ and were not correlated with the specific tip geometry (straight versus cantilevered). The large spread in observed behavior prevents quantitative analysis, but generally the magnitude of the changes decreased by approximately $30-60 \%$ in the aqueous environment.

The ability of the cantilevered NSOM probes to track small topography features on fragile samples in ambient and aqueous environments has been demonstrated. Comparison of images of lipid films taken in air and under water show that the lower tip resonance and the decrease of the $Q$ factor in water are not detrimental to the imaging performance. Moreover, investigation of the effects of drive amplitude on optical resolution reveals that by reducing the drive amplitude, the vertical oscillation of the tip can be maintained within the collimation region for the light exiting the aperture. This illustrates that optical resolution is not compromised in the cantilevered design utilizing tapping-mode force feedback. While considerably less efficient than conventional NSOM probes at delivering light to the end of the tip, the cantilevered probes are still capable of single molecule fluorescence detection limits. These features combine to extend the range of NSOM imaging capabilities, which promises to be a valuable tool for future investigations of biological systems.

The authors gratefully acknowledge the support of NSF (CHE-9612730), NSF-CAREER (CHE-9703009) and the Searle Scholars Program/The Chicago Community Trust.

${ }^{1}$ E. Betzig and R. J. Chichester, Science 262, 1422 (1993).

${ }^{2}$ X. S. Xie and R. C. Dunn, Science 265, 361 (1994).

${ }^{3}$ H. F. Hess, E. Betzig, T. D. Harris, L. N. Pfeiffer, and K. W. West, Science 264, 1740 (1994).

${ }^{4}$ M. A. Paesler and P. J. Moyer, Near-Field Optics: Theory, Instrumentation, and Applications (Wiley, New York, 1996).

${ }^{5}$ J. K. Trautman, J. J. Macklin, L. E. Brus, and E. Betzig, Nature (London) 369, 40 (1994).

${ }^{6}$ H. Muramatsu, N. Chiba, K. Homma, K. Nakajima, T. Ataka, S. Ohta, A. Kusumi, and M. Fujihira, Appl. Phys. Lett. 66, 3245 (1995).

${ }^{7}$ C. E. Talley, G. Cooksey, and R. C. Dunn, Appl. Phys. Lett. 69, 3809 (1996).

${ }^{8}$ S. Shalom, K. Lieberman, A. Lewis, and S. R. Cohen, Rev. Sci. Instrum. 63, 4061 (1992).

${ }^{9}$ C. W. Hollars and R. C. Dunn, J. Phys. Chem. 101, 6313 (1997).

${ }^{10}$ A. H. LaRosa, B. I. Yakobson, and H. D. Hallen, Appl. Phys. Lett. 67, 2597 (1995).

${ }^{11}$ M. Stahelin, M. A. Bopp, G. Tarrach, A. J. Meixner, and I. ZschokkeGranacher, Appl. Phys. Lett. 68, 2603 (1996).

${ }^{12}$ E. Kalb, S. Frey, and L. K. Tamm, Biochim. Biophys. Acta 1103, 307 (1992).

${ }^{13}$ Ch. Lienau, A. Richter, and T. Elsaesser, Appl. Phys. Lett. 69, 325 (1996).

${ }^{14}$ D. I. Kavaldijiev, R. Toledo-Crow, and M. Vaez-Iravani, Appl. Phys Lett. 67, 2771 (1995). 